\title{
METABOLISMO LIPÍDICO DURANTE O EXERCÍCIO FÍSICO: MOBILIZAÇÃO DO ÁCIDO GRAXO
}

\author{
Ellen Cristini de Freitas \\ Universidade de São Paulo, Ribeirão Preto, São Paulo, Brasil \\ Mariana Pereira Nobrega \\ Universidade Estadual Paulista "Júlio de Mesquita Filho", Araraquara, São Paulo, \\ Brasil
}

Flavia Rosa Troncom

Universidade de São Paulo, Ribeirão Preto, São Paulo, Brasil

Gabriel Silveira Franco

Universidade de São Paulo, Ribeirão Preto, São Paulo, Brasil

\begin{abstract}
Resumo
É sabido que os ácidos graxos são importante fonte de energia para contração muscular. As principais fontes metabólicas de energia derivadas dos lipídios são os ácidos graxos do tecido adiposo, os triglicerídeos intramusculares e os triglicerídeos circulantes do plasma. A contribuição de cada um depende do exercício realizado, duração, intensidade e estado de treinamento do indivíduo. O treinamento de longa duração provoca adaptações no organismo, como aumento da capacidade oxidativa dos músculos esqueléticos e maior participação dos lipídios como substrato energético durante o esforço, ocasionando possível melhora no desempenho do atleta. O objetivo do presente estudo foi investigar a regulação do metabolismo lipídico durante a realização do exercício físico.
\end{abstract}

Palavras-chave: Ácidos Graxos. Lipólise. Exercício Físico. Metabolismo.

\section{Introdução}

\begin{abstract}
A duas maiores fontes de energia utilizadas durante a realização Ado exercício físico são carboidratos (glicogênio e glicose) e lipídios (triglicerídeos). Embora aminoácidos de cadeia ramificada e alguns outros aminoácidos possam ser oxidados pelo músculo, sua contribuição é muito pequena quando comparada aos substratos energéticos inicialmente citados (JEUKENDRUP; SARIS; WAGENMAKERS, 1998a).

A relativa contribuição desses substratos na produção total de energia depende da intensidade e duração do esforço realizado. No entanto, durante período de repouso e realização de exercício de baixa
\end{abstract}


intensidade a moderado, os ácidos graxos de cadeia longa são os principais substratos energéticos participantes na produção de ATP (adenosina trifosfato) muscular (CLAVEL et al., 2002).

A prática regular de atividade física promove adaptações relevantes quanto a modificações no metabolismo lipídico, sugerindo o uso desse substrato energético como principal fonte de energia. Baseado nas citações acima, o objetivo do presente estudo foi investigar a mobilização dos ácidos graxos durante o exercício físico.

\section{Ácidos graxos como reserva de energia}

Os lipídios representam uma grande fonte de reserva corporal. Os ácidos graxos (AG), estocados na forma de triacilglicerol (TG) são reservas altamente concentradas de energia metabólica porque são reduzidos e anidros e representam a principal reserva energética disponível no homem. O rendimento da oxidação completa de ácidos graxos é em torno de $9 \mathrm{Kcal} / \mathrm{g}$, em contraste com cerca de $4 \mathrm{Kcal} / \mathrm{g}$ para glicídeos e proteínas. Os carboidratos são armazenados na presença de água ( $2 \mathrm{~g}$ de água por grama de glicogênio armazenado). Em razão disso, a capacidade de estocagem muscular e hepática de glicogênio é limitada. Porém, os lipídeos podem ser estocados no corpo em grandes quantidades, pelo menos 6 vezes mais energia do que $1 \mathrm{~g}$ de glicogênio hidratado. Portanto, lipídeos são combustíveis muito mais eficientes por unidade de peso (JEUKENDRUP; SARIS; WAGENMAKERS, 1998a).

No entanto, o principal fator problemático associado ao uso dos lipídios como combustível durante o esforço físico não é a disponibilidade física dos lipídios como fonte de energia, mas a velocidade na qual podem ser captados pelo músculo esquelético e oxidados para o fornecimento de energia. Isso demonstra que para uma oxidação aumentada de lipídios existe um padrão referencial quanto à intensidade e duração de esforço (AHLBORG et al., 1974).

\section{Mobilização de lipídios durante o exercício físico}

Os tipos de exercícios físicos que se beneficiam de forma significativa do metabolismo dos AG são aqueles com duração maior que 30 minutos e que se prolongam por algumas horas. Como já foi mencionado, a intensidade é um fator determinante na mobilização e utiliza- 
ção dos carboidratos e lipídios, visto que há uma relação direta entre a intensidade do esforço e a utilização de glicose como substrato (ROMIJN et al., 1993, 2000; BROOKS; MERCIER, 1994; BROOKS, 1997, 1998).

O exercício físico de baixa intensidade (25\% a $65 \% \mathrm{VO} 2 \mathrm{max})$ aumenta cerca de 5 a 10 vezes a oxidação de AGL quando comparado ao repouso, devido à alta demanda energética dos músculos ativos e disponibilidade dos AGL provenientes do tecido adiposo (WOLFE et al., 1990). Por sua vez, o exercício de intensidade moderada aumenta ainda mais o fluxo sanguíneo no tecido adiposo e causa aumento ainda maior deste no músculo esquelético (MARTIN et al.; 1993).

Os AGL que se desprendem do tecido adiposo ou aqueles derivados dos estoques de triacilgliceróis intramuscular (TGIM) ou ainda da hidrólise de lipoproteínas circulantes (QM e VLDL) representam os substratos energéticos preferidos para o metabolismo energético, sendo consequência de um processo denominado lipólise (CARLSON; EKELUND; FROBERG, 1971; HURLEY et al., 1986; KIENS, 2006).

Segundo GOODMAN (1970), o processo de lipólise no tecido adiposo ocorre durante a realização de exercício de baixa intensidade, sendo mediado pelo Sistema Nervoso Simpático (SNS), que estimula ação de hormônios, especialmente das catecolaminas (GOODMAN, 1970; WOLFE et al., 1990). Estando de acordo com essa afirmação, Kather e Simon (1977) acreditam que somente as catecolaminas podem estimular efetivamente o processo de lipólise. No entanto, encontra-se descrito na literatura que outros hormônios também participam na estimulação da lipólise, tais como hormônio de crescimento (GH), cortisol e hormônio estimulador da tireoide. Contudo, é consenso que a insulina apresenta um forte efeito inibitório sobre o processo de lipólise (GOODMAN, 1970; WOLFE et al., 1990).

As catecolaminas apresentam ação estimulatória via $\beta(\beta-1, \beta-2$ e $\beta 3$ adrenérgicos) que se acoplam com proteínas Gs (proteína $\mathrm{G}$ estimulatória), sendo que os receptores $\beta 3$ são os mais importantes para o processo de lipólise (KATHER; SIMON, 1977; FRANZ et al., 1983; WOLFE et al., 1990; CAREY, 1998)

Um dado interessante sobre esses receptores é que a responsividade de receptores $\beta$-adrenérgicos é elevada para catecolaminas no adipócito como resultado obtido na prática do exercício físico. Além disso, o treinamento físico aumenta ainda mais essa responsividade. Como consequência, há aumento na mobilização e concentração plas- 
mática de $\mathrm{AG}$, que é o passo determinante para sua oxidação no músculo esquelético. Foi demonstrado, contudo, que a velocidade de aparecimento de AG e glicerol no sangue a partir do tecido adiposo é reduzida no indivíduo altamente treinado (JEUKENDRUP; SARIS; WAGENMAKERS, 1998a, 1998b; HOROWITZ; KLEIN, 2000), sugerindo maior liberação de TGIM como fonte de combustível nesse estado treinado (MARTIN et al., 1993).

A ativação da lipólise (hidrólise de lipídios) por meio das catecolaminas é mediada por um aumento na concentração intracelular do monofosfato de adenosina cíclico (AMPc), desencadeando ativação da proteína quinase (PKA), que por sua vez irá fosforilar no adipócito a enzima hormônio lipase sensível (HSL), sendo esta a principal enzima envolvida na lipólise, bem como as pirilipinas. As pirilipinas são proteínas envolvidas com a superfície limitante de gotículas de lipídios presentes nos adipócitos, cuja função principal parece ser criar uma barreira limitando a ação da HSL. A fosforilação das perilipinas por meio da PKA libera esse impedimento, permitindo que a enzima hormônio lípase sensível fosforilada (HSP-P) realize sua ação na gotícula de gordura (CAREY, 1998; LONDOS et al., 1996; HOLM et al., 2000).

É sabido que a insulina exerce um efeito inibitório ao processo de lipólise, porém, durante o exercício físico, a concentração plasmática de insulina é reduzida, decorrente do efeito inibitório das catecolaminas sobre a liberação de insulina pancreática (WAHRENBERG et al., 1987; KIENS, 2006). De acordo com Arner et al. (1990), o efeito inibitório a lipólise observado pela presença da insulina possivelmente seja devido a uma redução na concentração de AMPc em função da estimulação da fosfodiesterase intracelular que degrada AMPc em AMP, dessa forma inibindo a estimulação da HLS.

A combinação desses efeitos estimulatórios a catecolaminas e inibitórios a insulina resulta no aumento do processo de lipólise no tecido adiposo durante exercício de baixa a moderada intensidade. Por outro lado, exercícios de alta intensidade ( $>80 \% \mathrm{VO} 2$ máx) aumentam na circulação fluxo glicolítico, concentração plasmática de lactato, podendo reduzir o índice de lipólise e aumentar reesterificação dos AG no tecido adiposo, podendo interferir na regulação da mobilização dos ácidos graxos (WAHRENBERG et al., 1987).

Porém, é importante salientar que o gasto energético proporcionado por atividade de alta intensidade é superior, mesmo que a fonte 
predominante não sejam os lipídios, gerando quantitativamente um aumento no uso de lipídios em comparação a atividades de baixa a moderada intensidade, pois o gasto energético total gerado pela atividade é maior quanto mais intensa a necessidade da demanda energética (ROMIJN et al., 1993).

O resultado final da lipólise consiste em três moléculas de ácidos graxos livres e uma molécula de glicerol, que devem ser transportadas do citosol das membranas celulares para a circulação sanguínea. A molécula de glicerol livre não pode ser reutilizada pelo tecido adiposo, pois esse tecido não contém quantidades significativas da enzima glicerol quinase. Sendo assim, os níveis de glicerol sanguíneo podem ser considerados uma medida indireta da taxa de lipólise no organismo. O glicerol será transportado ao fígado, onde será utilizado como um precursor da gliconeogênese (JEUKENDRUP; SARIS; WAGENMAKERS, 1998b).

Os AGL resultantes do processo de lipólise atravessam a membrana celular do adipócito de forma passiva ou são transportados por meio de proteínas. Foram identificadas recentemente proteínas ligadoras de ácidos graxos na membrana celular e no citossol, com o papel principal de facilitarem sua captação: proteína ligadora de ácidos graxos da membrana plasmática (FABPPM), a translocase de ácido graxo (FAT), a proteína transportadora de ácido graxo (FATP) e a proteína ligadora de ácidos graxos citossólica (FABPC) (LUIKEN et al., 1999; TURCOTTE, 2000).

Turcotte, Richter e Kiens (1992) mostraram que a captação de AGL no plasma, após 3 horas de exercício, é significativamente maior em homens treinados comparados a não treinados. Esses dados sugerem que a captação de AGL pelo músculo esquelético é mediada por um transportador saturável (TURCOTTE, 2000), tornando a captação limitada. No entanto, o treinamento físico pode aumentar a expressão gênica do RNA mensageiro (RNAm) dessas proteínas e proporcionar maior captação de ácidos graxos nos músculos ativos (TURCOTTE et al., 1999). Em seguida são liberados na corrente sanguínea, ligando-se às albuminas (SPECTOR, 1975).

A concentração plasmática de albuminas é constante (aproximadamente $6 \mathrm{mmol} / \mathrm{L}$ ), no entanto a concentração de ácidos graxos pode variar de 0,2 a $1,0 \mathrm{mmol} / \mathrm{L}$. Isso demonstra uma grande capacidade de transporte de ácidos graxos via albumina em condições fisiológicas. Durante exercício de intensidade moderada a concentração de ácidos 
graxos pode aumentar em até 20 vezes. Porém, cada molécula de albumina apresenta quantidade finita de receptores para a ligação de ácidos graxos. Se ocorrer saturação do complexo albumina-ácidos graxos, a taxa de ligação e de transporte estará diminuída, contribuindo para o aumento da taxa de reesterificação (SPECTOR, 1975; MAUGHAN; GLEESON; GREENHAFF, 2000).

Apesar de a principal reserva de lipídios do corpo estar localizada no tecido adiposo, quantidades de TG são armazenadas no músculo esquelético como triacilglicerol intramuscular (TGIM). Uma parte pode estar contida nas células adiposas dispersas entre as fibras musculares do tecido (intermuscular), mas também existem evidências, pela microscopia luminosa e eletrônica, da existência de gotículas de triacilglicerol localizadas próximo às mitocôndrias no interior das fibras (intramuscular) (MAUGHAN; GLEESON; GREENHAFF, 2000).

Embora pouco se saiba sobre o papel do estoque TG inter - versus - intramuscular, o fato é que sujeitos treinados apresentam mais TGIM e menos intermuscular (HOPPELER, 1986) e oxidam mais TGIM (MARTIN et al., 1993) sugerindo que os TGIM são mais importantes em termos de provisão de energia.

Estudos histológicos revelam que o tipo de fibra I contém mais TGIM do que o tipo de fibra IIa em seres humanos. O tipo de fibra IIb indicou a menor concentração de TGIM (ESSEN, 1977). Além disso, o próprio treinamento físico faz que a deposição de TG seja diferente entre os tipos de fibras musculares (ALBERNETHY; THAYER; TAYLOR, 1990).

Romijn et al. (1993) investigaram a captação de ácidos graxos e estimaram a utilização de TGIM usando isótopos estáveis. Encontraram que durante exercício de baixa intensidade ( $25 \%$ do VO2máx) a contribuição dos TGIM é mínima (7\%). Os ácidos graxos plasmáticos e a glicose parecem ser os substratos mais importantes nessa intensidade, porém a gordura é o combustível predominante. Em exercícios de intensidade moderada (65\% do VO2máx) TGIM e glicogênio muscular parecem ser os substratos mais importantes. Os TGIM foram oxidados em altas taxas nessa intensidade de esforço (26\%); no entanto, os ácidos graxos plasmáticos foram utilizados em menor taxa comparandose ao exercício de baixa intensidade. Quando a intensidade do exercício foi aumentada (85\% VO2máx.) a contribuição dos ácidos graxos plasmáticos foi ainda menor, e a oxidação do TGIM também foi redu- 
zida (8\%), sugerindo que a utilização ótima dos TGIM está entre $25 \%$ e $85 \%$ VO2máx.

Segundo Horowitz e Klein (2000), durante a realização de exercício de baixa intensidade a oxidação de AG é derivada dos AG plasmáticos. Com o aumento da intensidade, a relativa contribuição dos TGIM aumenta e pode representar quase a metade de todo lipídio oxidado. Estando de acordo com essa citação, Albernethy, Thayer e Taylor (1990) descrevem que a reserva mais importante de ácidos graxos não plasmático disponível para oxidação durante exercício moderado e prolongado são os TGIM.

Em contrapartida, exercícios de alta intensidade (85\% VO2max.) promovem relativamente altos padrões de esgotamento de glicogênio muscular. A participação do metabolismo dos AGL fica sensivelmente diminuída, sendo essa intensidade predominantemente anaeróbia glicolítica (COYLE et al., 1995).

Vários autores descrevem que músculos treinados estocam mais lipídeos intramusculares, que se localizam ao longo da superfície do sistema mitocondrial, o qual pode, teoricamente, elevar a capacidade de suprimento e oxidação de ácidos graxos derivados dos estoques intracelulares. $\mathrm{O}$ aumento nos estoques intracelulares de TG indica que indivíduos treinados, especialmente em treinamentos aeróbios, utilizam mais os estoques TGIM que os de ácidos graxos sanguíneos. A vantagem disso reside no fato de que algumas barreiras existentes no processo de oxidação do ácido graxo circulante, como o transporte dos ácidos graxos no plasma e sua passagem da célula muscular, não são necessários e, portanto, a sua oxidação é rapidamente desencadeada (HURLEY et al., 1986; MARTIN et al., 1993; ROMIJN et al., 1993; HOROWITZ; KLEIN, 2000; ROEPSTORFF; VISTISEN; KIENS, 2005).

Outra fonte potencial de AGL para o músculo provém dos TG ligados às lipoproteínas (VLDL e QM). No plasma essas lipoproteínas são degradadas pela enzima lipase lipoproteica (LPL) (HAVEL; PERNOW; JONES, 1967).

Somente após a hidrólise dos TG presentes nas Lp por meio da LPL, os AGL poderão ser transportados para a célula muscular (BRAUN; SEVERSON, 1992). A degradação de Lp ricas em TG com liberação de ácidos graxos e glicerol é uma consequência da ação da LPL, uma enzima localizada na superfície endotelial no músculo e tecido adiposo (NILSSON-EHLE; GARFINKEL; SCHOTZ, 1980). 
Nesses locais ela está ligada aos proteoglicanos de sulfato de heparina. Assim, uma característica da enzima é sua capacidade de se ligar à heparina, o que possibilita a sua dosagem após a injeção de heparina no plasma (GROOT; SHEEK, 1984). A enzima só é ativa na sua forma dimérica, sendo que a presença de apoCII é essencial para a sua ativação. No processo de hidrólise os AGL resultantes são captados pelos tecidos periféricos para a produção ou reserva de energia. Simultaneamente, o excesso de componentes na superfície dos QM e VLDL é transferido para a HDL, juntamente com apoCII (GROOT; SHEEK, 1984). Portanto, a LPL é essencial para a regulação de TG séricos para o armazenamento de TG nos adipócitos e para o fornecimento de combustível celular.

A atividade da LPL parece elevar os níveis do colesterol da HDL circulante. Foi observada uma correlação positiva da atividade da LPL pós heparina com HDL-C após exercício (KANTOR et al., 1984; SADY et al., 1986).

Foi também mostrado que o nível da atividade LPL é quase duas vezes maior após $42 \mathrm{~km}$ de corrida, demonstrando que o exercício intenso prolongado aumenta agudamente a atividade da enzima (SADY et al., 1986).

Todo aumento no consumo de ácidos graxos dos triglicerídeos plasmáticos (AGTP) pelo músculo esquelético durante o exercício é acompanhado presumivelmente pelo aumento na atividade da LPL. No entanto, Borensztajn et al. (1975) descrevem que os níveis de atividade LPL diferem consideravelmente entre os três tipos de fibra muscular esquelética.

Há um acréscimo no nível de LPL em fibras vermelhas de contração lenta, com nível intermediário em fibras vermelhas de contração rápida e níveis extremamente baixos em fibras brancas de contração rápida (GOLLNICK et al., 1972; BALDWIN et al., 1973).

As fibras musculares vermelhas são preferencialmente recrutadas durante trabalho de intensidade baixa a moderada por períodos prolongados de tempo (GOLLNICK et al., 1972; BALDWIN et al., 1973).

Segundo Baldwin et al. (1973), no músculo esquelético a maior porção de AGTP é apanhada pelas fibras vermelhas de contração lenta e rápida; em contraste, as fibras brancas de contração rápida têm capacidade muito reduzida em aprisionar ácidos graxos plasmáticos. Essa interpretação é condizente com a observação de que dos três tipos de 
fibras somente as fibras brancas de contração rápida têm uma baixa capacidade oxidativa.

No entanto, acredita-se que a contribuição dos TG plasmáticos seja muito pequena em relação à produção de energia durante o exercício, possivelmente menos que 5\% (Romijn et al., 1993). Sugere-se que o fator mais importante esteja relacionado à influência positiva no perfil das Lp plasmáticas após exercício físico (GORSKI; OSCAI; PALMER, 1990).

\section{Conclusões}

1. A mobilização dos AGL a partir do tecido adiposo é dependente da taxa de lipólise, da capacidade de transporte dos AGL pelo plasma e da taxa de reesterificação destes pelos adipócitos.

2. Durante a realização do exercício físico ocorre aumento da taxa de lipólise no tecido adiposo, especialmente no treinamento aeróbio, que resulta em aumento significativo na oxidação de AGL, no volume mitocondrial, assim como na atividade de suas enzimas.

3. O treinamento também induz a um aumento na densidade capilar no tecido muscular, proporcionando aumento na superfície de trocas entre o sangue e o tecido muscular, o fluxo sanguíneo e consequentemente a oferta de $\mathrm{O} 2$ e ácidos graxos para fibra muscular.

4. A insulina apresenta ação inibitória por meio de receptores $\alpha_{2}$ adrenérgicos, os quais estão ligados à proteína $\mathrm{G}$ inibitória (PGi), inibindo a formação do AMPc e consequentemente o processo de lipólise (ARNER et al., 1990).

5. Observa-se que durante o exercício a concentração plasmática de insulina é reduzida, decorrente do efeito inibitório das catecolaminas sobre a liberação de insulina pancreática, apresentando este um potente efeito inibitório da lipólise. O efeito inibitório da lipólise observado pela presença da insulina possivelmente seja devido a uma redução na concentração de AMPc em função da estimulação da fosfodiesterase intracelular que degrada AMPc em AMP, dessa forma inibindo a estimulação da LHS.

Lipid metabolism during exercise: mobilization of fatty acid

\section{Abstract}

It is known for a long time that fatty acids are important energy source for muscle

Pensar a Prática, Goiânia, v. 15, n. 3, p. 551-820, jul./set. 2012 
contraction. The main sources of metabolic energy derived from lipids are free fatty acids from adipose tissue and intramuscular triglycerides circulating plasma triglycerides. The contribution of each one depends on the type of exercise done, duration, intensity and state of training of the individual. The endurance training provokes adaptations in the organism as, increase of the oxidative capacity of the skeletal muscles and, consequently bigger participation of the lipids as energetic substrate during realization of the effort, possibly causing an improvement in performance of the athlete. The aim of the present study was to investigate regulation of the lipids metabolism, during the physical exercise.

Keywords: Fatty Acids. Lipolysis. Physical Exercise. Metabolism.

\section{Metabolismo de los lípidos durante el ejercicio: movilización del ácido graso}

\section{Resumen}

Se sabe que los ácidos grasos son fuente importante de energía para la contracción muscular. Las principales fuentes de energía metabólica derivada de los lípidos son los ácidos grasos del tejido adiposo intramuscular y triglicéridos plasmáticos de triglicéridos circulantes. La contribución de cada uno depende del ejercicio realizado, duración, intensidad y nivel de entrenamiento del individuo. La formación a largo plazo provoca adaptaciones en el cuerpo, como aumento de la capacidad oxidativa del músculo esquelético y una mayor participación de los lípidos como sustrato energético durante el ejercicio, dando lugar a una posible mejora en el rendimiento deportivo. El propósito de este estudio fue investigar la regulación del metabolismo de los lípidos durante el curso del ejercicio.

Palabras clave: Ácidos Grasos. La Lipólisis. El Ejercicio. El Metabolismo.

\section{Referências}

AHLBORG, G. et al. Substrate turnover during prolonged exercise in man: splanchnic and leg metabolism of glucose, free fatty acids, and amino acids. J. Clin Invest., v. 53, n. 4, p. 1080-1090, 1974.

ALBERNETHY, P. J.; THAYER, R.; TAYLOR, A. W. Acute and chronic responses of skeletal muscle to endurance and sprint exercise: a review. Sports Med., v. 10, n. 6, p. 365-389, 1990.

ARNER, P. et al. Adrenergic regulation of lipolysis in situ at rest and during exercise. J. Clin. Invest., v. 85, n. 3, p. 893-898, 1990.

BALDWIN, K. M. et al. Substrate depletion in different types of muscle and in liver during prolonged running. Am. J. Physiol., v. 225, n. 5, p. 1045-1050, 1973. 
BORENSZTAJN, J. et al. Effect of exercise on lipoprotein lipase activity in rat heart and skeletal muscle. Am. J. Physiol., v. 229, n. 2, p. 394-397, 1975.

BRAUN, J. E.; SEVERSON, D. L. Regulation of the synthesis, processing and translocation of lipoprotein lipase. Biochem. J., v. 287, p. 337-347, 1992.

BROOKS, G. A. Importance of the 'crossover' concept in exercise metabolism. Clin. Exp. Pharmacol. Physiol., v. 24, n. 11, p. 889-895, 1997.

BROOKS, G. A. Mammalian fuel utilization during sustained exercise. Comp. Biochem. Physiol. B. Biochem. Mol. Biol., v. 120, n. 1, p. 89-107, 1998.

BROOKS, G. A.; MERCIER, J. Balance of carbohydrate and lipid utilization during exercise: the 'crossover' concept. J. Appl. Physiol., v. 76, n. 6, p. 2253-2261, 1994.

CAREY, G. B. Mechanisms regulating adipocyte lipolysis. Adv. Exp. Med. Biol., v. 441, p. 157-170, 1998.

CARLSON, L. A.; EKELUND, L. G.; FROBERG, S. O. Concentration of triglycerides, phospholipids and glycogen in skeletal muscle and of free fatty acids and beta-hydroxibutyric acid in blood in man in response to exercise. Eur. J. Clin. Invest., v. 1, n. 4, p. 248-254, 1971.

CLAVEL, S. et al. Effect of endurance training and/or fish oil supplemented diet on cytoplasmic fatty acid binding protein in rat skeletal muscles and heart. Eur. J. Appl. Physiol., v. 87, n. 3, p. 193-201, 2002.

COYLE, E. F. Substrate utilization during exercise in active people. Am. J. Nutr., v. 6, n. (4 suppl), p. 968S-979S, 1995.

ESSÉN, B. Intramuscular substrate utilization during prolonged exercise. Ann. N Y Acad. Sci., v. 301, p. 30-44, 1977.

FRANZ, I. W. et al. Aspects of hormonal regulation of lipolysis during exercise: effects of chronic beta-receptor blockade. Int. J. Sports Med., v. 4, n. 1, p. 14-20, 1983. 
GOLLNICK, P. D. et al. Diet, exercise and glycogen changes in human muscle fibers. J. Appl. Physiol., v. 33, n. 4, p.421-425, 1972.

GOODMAN, H. M. Permissive effects of hormones on lipolysis. Endocrinology., v. 86, n. 5, p. 1064-1074, 1970.

GORSKI, J.; OSCAI, L. B.; PALMER, W. K. Hepatic lipid metabolism in exercise and training. Med. Sci. Sports Exerc., v. 22, n. 2, p. 213-221, 1990.

GROOT, P. H.; SHEEK, L. M. Effects of fat ingestion on high density lipoprotein profiles in human sera. J. Lipid Res., v. 25, n. 7, p. 684692, 1984.

HAVEL, R. J.; PERNOW, B.; JONES, N. L. Uptake and release of free fatty acids and other metabolites in the legs of exercising men. $\mathbf{J}$. Appl. Physiol., v. 23, n. 1, p. 90-99, 1967.

HOLM, C. et al. Molecular mechanisms regulating hormone-sensitive lipase and lipolysis. Annu. Rev. Nutr., v. 20, p. 365-393, 2000.

HOPPELER, H. Exercise-induced ultrastructural changes in skeletal muscle. Int. J. Sports Med., v. 7, n. 4, p. 187-204, 1986.

HOROWITZ, J. F.; KLEIN, S. Lipid metabolism during endurance exercise. Am. J. Clin. Nutr., v. 72, n. (2 suppl), p. 558S-563S, 2000.

HURLEY, B. F. et al. Muscle triglyceride utilization during exercise: effect of training. J. Appl. Physiol., v. 60, n. 2, p. 562-567, 1986.

JEUKENDRUP, A. E.; SARIS, W. H. M.; WAGENMAKERS, A. J. M. Fat metabolism during exercise: a review. Part I: Fatty acid mobilization and muscle metabolism. Int. J. Sports Med., v. 19, n. 4, p. 231-244, 1998a.

JEUKENDRUP, A. E.; SARIS, W. H. M.; WAGENMAKERS, A. J. M. Fat metabolism during exercise: a review. Part II: Regulation of metabolism and effects of training. Int. J. Sports Med., v. 19, n. 5, p. 293-302, 1998b.

KANTOR, M. A. et al. Acute increase in lipoprotein lipase following prolonged exercise. Metabolism, v. 33, n. 5, p. 454-457, 1984. 
KATHER, H.; SIMON, B. Catecholamine-sensitive adenylate cyclase of human fat cell ghosts: a comparative study using different betaadrenergic agents. Metabolism, v. 26, n. 11, p. 1179-1184, 1977.

KIENS, B. Skeletal muscle lipid metabolism in exercise and insulin resistence. Physiol. Rev., v. 86, n. 1, p. 205-243, 2006.

LONDOS, C. et al. Perilipin: a possible roles in structure and metabolism intracellular neutral lipids in adipocytes and steroidogenic cells. Int. J. Obes. Relat. Metab. Disord., v. 20, n. suppl 3, p. S97-101, 1996.

LUIKEN, J. J. et al. Cellular fatty acid transport in heart and skeletal muscle as facilitated by proteins. Lipids, v. 34, n. suppl: 169S-175S, 1999.

MARTIN, W.H. 3rd et al. Effect of endurance training on plasma free fatty acid turnover and oxidation during exercise. Am. J. Physiol., v. 265, p. E708-E714, 1993.

MAUGHAN, R.; GLEESON, M.; GREENHAFF, P. L. Bioquímica do exercício e do treinamento. São Paulo: Manole, 2000.

NILSSON-EHLE, P.; GARFINKEL, A. S.; SCHOTZ, M. C. Lipolytic enzymes and plasma lipoprotein metabolism. Annu. Rev. Biochem., v. 49, p. 667-693, 1980.

ROEPSTORFF, C.; VISTISEN, B.; KIENS, B. Intramuscular triacylglycerol in energy metabolism during exercise in humans. Exerc. Sport Sci. Rev., v. 33, n. 4, p. 182-188, 2005.

ROMIJN, J. A. et al. Regulation of endogenous fat and carbohydrate metabolism in relation to exercise intensity and duration. Am. J. Physiol., v. 265, p. E380-E391, 1993.

ROMIJN, J. A. et al. Substrate metabolism during different exercise intensities in endurance-trained women. J. Appl. Physiol.,v. 88, n. 5, p. 1707-1714, 2000.

SADY, S. P. et al. Prolonged exercise augments plasma triglyceride clearance . J. Am. Med. Assoc., v. 256, n. 18, p. 2552-2555, 1986. 
SPECTOR, A. A. Fatty acid binding to plasma albumin. J. Lipid Res., v. 16, n. 3, p. 165-179, 1975.

TURCOTTE, L. P. Muscle fatty acid uptake during exercise: possible mechanisms. Exerc. Sports Sci. Rev., v. 28, n. 1, p. 4-9, 2000.

TURCOTTE, L. P.; RICHTER, E. A.; KIENS, B. Increased plasma FFA uptake and oxidation during prolonged exercise in trained vs. untrained humans. Am. J. Physiol., v. 262, p. E791-E799, 1992.

TURCOTTE, L. P. et al. Training-induced elevation in FABP(PM) is associated with increase palmitate use in contrating muscle. J. Appl. Physiol., v. 87, n. 1, p. 285-293, 1999.

WAHRENBERG, H. et al. Acute adaptation in adrenergic control of lipolysis during physical exercise in human. Am. J. Physiol., v. 253, p. E383-E390, 1987.

WOLFE, R. R. et al. Role of triglyceride-fatty acid cycle in controlling fat metabolism in humans during and after exercise. Am. J. Physiol., v. 258, p. E382-E389, 1990.

Recebido em: 19/09/2011

Revisado em: 14/10/2011

Aprovado em: 22/11/2011

\section{Endereço para correspondência}

ecfreitas@yahoo.com

Ellen Cristini de Freitas

Universidade de São Paulo

Escola de Educação Física e Esporte de Ribeirão Preto.

Av: Bandeirantes, 3900

Monte Alegre

14040-900 - Ribeirao Preto, SP - Brasil 